\title{
Medición del Índice de Desarrollo del Hábitat (IDeHa) de Aguascalientes [México]
}

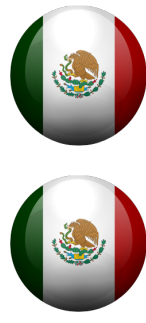

\section{Mauricio Ruiz Morales}

Director de Carrera de Arquitectura en Tecnológico de Monterrey. Doctorando en Ciencias de los Ámbitos Antrópicos [Universidad Autónoma de Aguascalientes]. Ciudad de México [DF] México. <arq.mauricio.ruiz@gmail.com>

\section{Alejandro Acosta Collazo}

Doctor en Arquitectura por la Universidad Nacional Autónoma de México. Profesor-Investigador en la Universidad Autónoma de Aguascalientes, Centro de Ciencias del Diseño y de la Construcción, Departamento de Diseño del Hábitat. Aguascalientes [Aguascalientes] México. <aacosta@correo.uaa.mx>

\section{Resumen}

En la actualidad, es necesario incluir variables multidimensionales para poder medir la calidad de vida y el índice de desarrollo de un sector poblacional. Por lo anterior, el presente artículo pretende mostrar una forma innovadora para medir el desarrollo del hábitat humano a partir de la consideración de variables poblacionales, de la vivienda y de la conectividad que pueden tener los habitantes de un sector determinado en una ciudad. El presente artículo muestra la metodología para la construcción del Índice de Desarrollo del Hábitat (IDeHa) y presenta una medición para tres sectores representativos de la ciudad de Aguascalientes.

\section{Palabras clave}

Índice de Desarrollo del Hábitat. Aguascalientes. Pobreza. Desarrollo sustentable. Calidad de vida.

\section{Measurement of Habitat Development Index (IDeHa) for Aguascalientes [Mexico]}

\begin{abstract}
At present, it is essential to include multidimensional variables to measure the quality of life and the growth rate of a population sector. Therefore, this article shows an innovative way to measure the development of the human habitat. The measurement is done considering variables of population, housing and connectivity of a specific sector in a city. This paper shows the methodology for the construction of Habitat Development Index (IDeHa) and shows a measurement for three representative areas of the city of Aguascalientes.
\end{abstract}

\section{Keywords}

Habitat Development Index. Aguascalientes. Poverty. Sustainable development. Quality of life. 


\section{Introducción}

De acuerdo a diversos factores, la situación de bienestar de las diferentes familias mexicanas, ha sufrido un cambio importante en el nivel cualitativo y cuantitativo por diferentes factores asociados al consumo, ingreso per capita, densidad poblacional y en términos generales a las nuevas condiciones del estilo de vida de las ciudades contemporáneas.

El crecimiento urbano obedece a una necesidad de mercado que se determina por: a) la factibilidad del negocio medida por el desarrollador inmobiliario, b) por la potencia y aceptación por parte del mercado y c) por el impulso y regulación por parte gubernamental. Así, en algunas de la ciudades de tamaño medio en el país, la implementación de nuevos fraccionamientos y su respectivo equipamiento, tienen ciertas características que buscan que el mercado considere deseable la compra de vivienda aunque no tengan las mejores condiciones de habitabilidad.

El crecimiento urbano, al responder en una medida importante a las expectativas de un mercado determinado, ha adquirido ciertas propiedades que no necesariamente ofrecen una mejor calidad de vida a los usuarios. De la misma manera en que se han cambiado ciertos valores en la edificación de nueva vivienda, también se han cambiado ciertas relaciones y referencias de zonas habitadas que se han construido en las décadas pasadas.

Éstas transformaciones en la ciudad han provocado que algunos sectores se encuentren con mayor riesgo o vulnerabilidad para considerarse en pobreza o marginación.

Las causas que originan la pobreza y la marginación tienen un carácter multifactorial y una relación de complejidad e interrelación alta, sin embargo, hay ciertos factores recurrentes. Por lo anterior, se torna fundamental analizar diferentes aspectos y combinaciones de factores para seleccionar los que tienen mayor relevancia en la determinación de la pobreza y marginación asociada a la vivienda.

Por lo anterior, dentro del presente artículo se explora una forma innovadora de analizar las condiciones de una vivienda o de un sector urbano a partir de un índice. El planteamiento contiene variables identificadas y agrupadas en 3 categorías.

La propuesta se denomina Índice de Desarrollo del Hábitat (IDeHa). Se explica a continuación.

\section{Bases para la construcción del Índice de Desarrollo del Hábitat}

Considerando diferentes perspectivas para encontrar un mejor equilibrio en la forma de producción de comunidades, se han incorporado diferentes corrientes de pensamiento para construir una herramienta que sirva para medir el hábitat y consecuentemente, obtener resultados objetivos y encontrar alternativas para potenciar la intervención efectiva y sustentable de un entorno edificado, mejorando sus condiciones de habitabilidad.

El Índice de Desarrollo del Hábitat es una herramienta que sirve para medir la calidad del Hábitat.

A pesar de existir diferentes formas de medir la situación de la vivienda en México y en Latinoamérica, no hay una herramienta específica para asociar el desarrollo de la calidad del hábitat con los conceptos de desarrollo escala humana (Max Neef, 1998: 50), de mejora de sociedades líquidas (Bauman, 2013: 21) y del decrecimiento (Latouche, 2009: 107). Así, el presente artículo pretende mostrar el IDeHa como una herramienta que mide las condiciones de unas zonas determinadas de una ciudad, desde una postura que incluye los conceptos de Desarrollo Sustentable como punto de partida para la medición.

La herramienta se puede utilizar en la medición del desarrollo del hábitat de diferentes localidades del territorio nacional. En el presente artículo se realiza un ensayo del IDeHa en la ciudad de Aguascalientes. 


\subsection{Variables y relaciones fundamentales en el desarrollo del hábitat}

Desde la década de los 70 del siglo pasado, diferentes pensadores han incursionado en nuevas estrategias y planteamientos para consolidar la idea de desarrollo del entorno humano o del desarrollo del hombre contemplando diferentes variables, estructuras y planteamientos socio económicos desde una realidad cambiante.

La sociedad se encuentra en un gran cambio de valores que por diversas causas han privilegiado al consumo y a los ideales del consumo como un motor para el desarrollo económico y aspiración de la sociedad.

La constitución de una nueva sociedad líquida (Bauman, 1999: 129), produce que la ciudades se vayan transformando y que tengan cada vez más componentes para el intercambio comercial, reduciendo de forma simultánea las posibilidades de cubrir diferentes satisfactores humanos (MaxNeef, 1993: 40). Por lo tanto, se reducen ciertos alcances en un habitante con un potencial de consumo bajo, es decir, el sector pobre tiene menos posibilidades para cubrir los satisfactores básicos a través de las relaciones de vivienda y ciudad.

A continuación se explican nuestras razones:

1. Una sociedad líquida tiene como fundamento la direccionalidad por sector en las formas de presentar la cultura, los deseos y satisfactores hacia el consumo. Así, el continuo consumo es una de las acciones principales, porque para poder generar transformaciones rápidas, aunque sea en imagen, es necesario cambiar rápidamente las formas de los objetos y de la forma de representación. Esto no implica que necesariamente se tenga que cambiar el fin último de la utilidad de un objeto, sin embargo, a través de un consumo acelerado, la población tiene la percepción de que hay una mejora y progreso porque se busca transmitir ciertos aspectos novedosos a las nuevas estructuras que se muestran a través de los objetos y discursos líquidos.

2. En la búsqueda de la representación nueva de una ciudad basada en la sociedad líquida, se reducen y comprimen espacios destinados a la población de todos los sectores socioeconómicos y se sustituyen por entornos que propician un consumo alto, definido por sector y con un impulso hacia el aumento de la frecuencia en el consumo. Lo anterior va en detrimento de la calidad de vida, sentido de comunidad y diversidad de acciones que pueden tener los habitantes de un sector de una ciudad que tenga equipamiento y servicios deficientes.

3. Adicionalmente, los servicios que el gobierno tiene que dotar a cada uno de los habitantes de una ciudad se ven comprometidos por el rápido crecimiento de nuevos fraccionamientos.

4. Los transportes públicos y los sistemas destinados a la comunicación de los habitantes, también se encuentran comprometidos por el rápido crecimiento urbano y por una defectuosa relación entre la planeación de la ciudad y entre la creación de nuevos fraccionamientos orientados a crear una relación adecuada con las actividades de los habitantes.

\section{2. Índice de Desarrollo del Hábitat. Construcción del modelo}

La presente herramienta, vincula las condiciones elementales del nivel de pobreza multidimensional (CONEVAL) y vulnerabilidad, manifestadas en el hábitat a través de tres niveles o dimensiones. Éstos niveles se conocen como dimensiones contextuales porque abarcan las características generales que se pretenden medir a partir de la herramienta del índice.

El siguiente nivel de aproximación se conoce como: la forma de medición del contexto y vivienda que abarca las cualidades específicas que se miden de la dimensión contextual. El componente operativo para encontrar los factores específicos de la medición, se denominan como indicadores, para medir la intensidad del desarrollo de vivienda y contexto; éstos, son datos específicos obtenidos de fuentes documentales confiables.

A partir de la estructura del índice, se genera una medición de diferentes puntos de una ciudad 
para poder obtener una referencia de medición. También se pueden comparar diferentes períodos en un mismo sector urbano.

Las dimensiones contextuales se muestran a continuación. Asimismo, se explica la forma de medición para la vivienda y el contexto, y la intensidad del indicador de cada una de las formas de medición. La fuente que se utiliza para la obtención de los diferentes datos en el Índice de Desarrollo del Hábitat, es el Instituto Nacional de Estadística y Geografía (INEGI):

\subsubsection{Población:}

1. Porcentaje de población de 15 años y más analfabeta: Personas de 15 a 130 años de edad que no saben leer ni escribir por cada 100 personas, de 15 a 130 años de edad.

2. Porcentaje de población de 15 años y más con educación básica incompleta: Personas de 15 a 130 años de edad que tienen como máxima escolaridad algún grado en primaria, algún grado en técnico o comercial con antecedente de primaria o uno o dos grados aprobados en secundaria por cada 100 personas de 15 a 130 años de edad.

3. Porcentaje de población de 25 años y más con al menos un grado aprobado en educación superior: Personas de 25 a 130 años de edad que tienen como máxima escolaridad algún grado aprobado de estudios técnicos con antecedente de preparatoria, profesional, normal superior y los de maestría o doctorado por cada 100 personas de 25 a 130 años de edad.

4. Porcentaje de población con limitación en la actividad derecho habiente a servicios de salud: Personas que tienen alguna dificultad para el desempeño y/o realización de tareas básicas de la vida cotidiana, que tienen derecho a recibir servicios médicos en alguna institución de salud pública o privada como resultado de una prestación laboral por ser pensionado o jubilado, por inscribirse o adquirir un seguro médico o por ser familiar designado beneficiario en: el Instituto Mexicano del Seguro Social (IMSS), el Instituto de Seguridad y Servicios Sociales de los Trabajadores del Estado (ISSSTE e ISSSTE estatal), Petróleos Mexicanos (PEMEX), la Secretaría de la Defensa Nacional (SEDENA), la Secretaría de Marina Armada de México (SEMAR), el Sistema de Protección Social en Salud o en otra por cada 100 personas con discapacidad.

5. Porcentaje de población con discapacidad: Personas que tienen dificultad para el desempeño y/o realización de tareas en la vida cotidiana por cada 100 personas.

6. Razón de dependencia total: Número de personas menores de 15 años y mayores de 64 años por cada 100 personas de 15 a 64 años de edad.

\subsubsection{Vivienda:}

1. Porcentaje de viviendas particulares habitadas con un dormitorio: Número de viviendas particulares habitadas con un dormitorio por cada cien viviendas particulares habitadas.

2. Porcentaje de viviendas particulares habitadas con un solo cuarto: Número de viviendas particulares habitadas con un solo cuarto por cada cien viviendas particulares habitadas.

3. Porcentaje de viviendas particulares habitadas con piso de tierra: Número de viviendas particulares habitadas con piso de tierra por cada cien viviendas particulares habitadas.

4. Porcentaje de viviendas particulares habitadas con más de 3 ocupantes por cuarto: Número de viviendas particulares habitadas con más de 3 ocupantes por cuarto, por cada cien viviendas particulares habitadas.

5. Porcentaje de viviendas particulares habitadas que disponen de luz eléctrica: Número de viviendas particulares habitadas que disponen de luz eléctrica por cada cien viviendas particulares habitadas.

6. Porcentaje de viviendas particulares habitadas que disponen de agua entubada en el ámbito de la vivienda: Número de viviendas particulares habitadas que disponen de agua entubada en el ámbito de la vivienda por cada cien viviendas particulares habitadas.

7. Porcentaje de viviendas particulares habitadas que disponen de drenaje: Número de viviendas particulares habitadas que disponen de drenaje por cada cien viviendas particulares habitadas.

8. Porcentaje de viviendas particulares habitadas que no disponen de refrigerador ni lavadora: Número de viviendas particulares habitadas que no disponen de refrigerador ni lavadora por cada cien viviendas particulares habitadas. 


\subsubsection{Conectividad:}

1. Porcentaje de viviendas particulares habitadas sin línea telefónica ni teléfono celular: Número de viviendas particulares habitadas sin línea telefónica ni teléfono celular por cada cien viviendas particulares habitadas.

2. Porcentaje de viviendas particulares habitadas que disponen de internet: Número de viviendas particulares habitadas que disponen de internet por cada cien viviendas particulares habitadas.

3. Porcentaje de viviendas particulares habitadas que disponen de automóvil o camioneta: Número de viviendas particulares habitadas que disponen de automóvil o camioneta por cada cien viviendas particulares habitadas.

A continuación se muestra el esquema propuesto para obtener el Índice de Desarrollo del Hábitat (Figura 1):

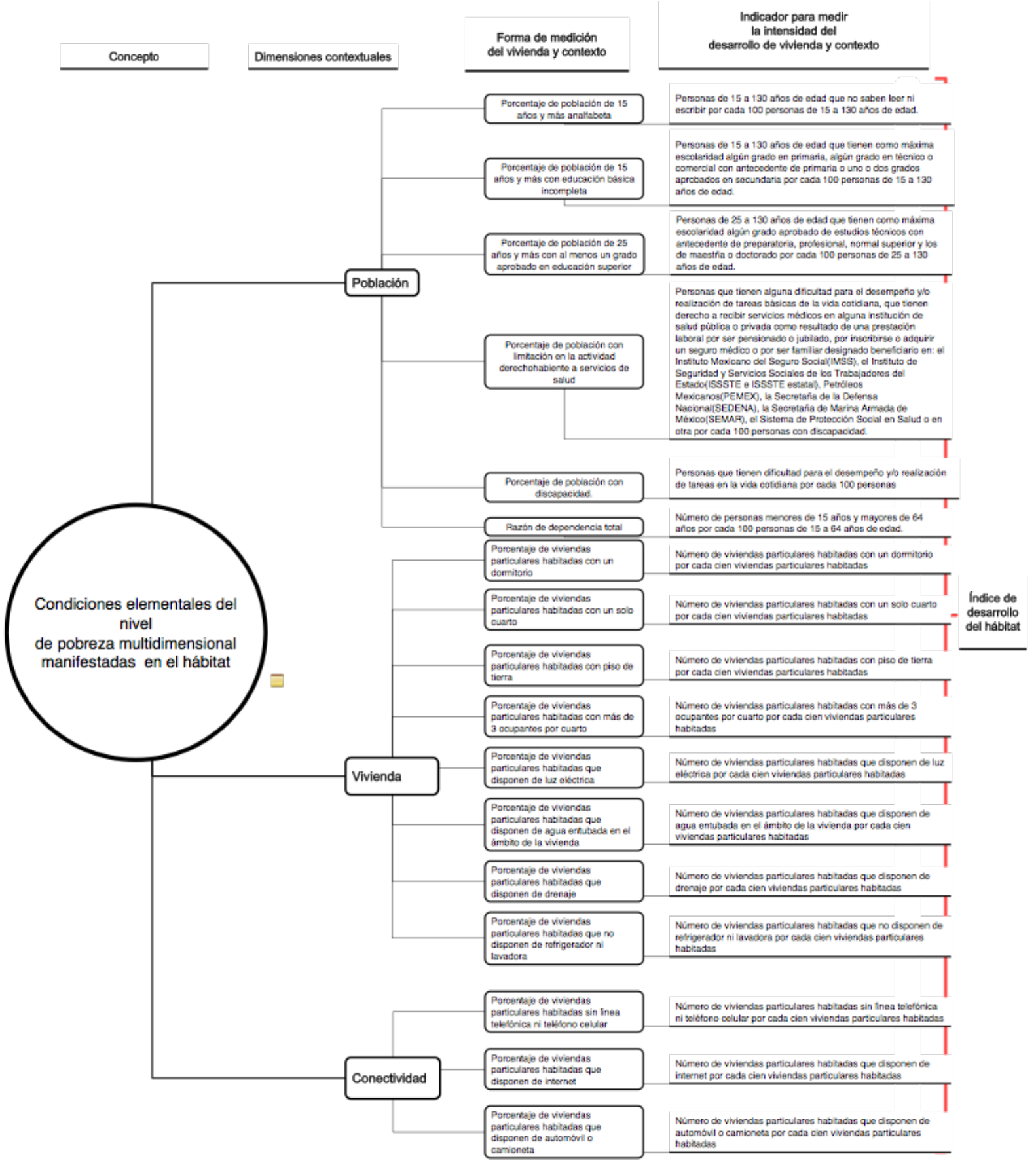

Figura 1. Esquema de Índice de Desarrollo del Hábitat. Elaboración propia con información del INEGI (2010). 


\section{Medición del Índice de Desarrollo del Hábitat en Aguascalientes}

Como se mencionó con anterioridad, la herramienta se puede aplicar para medir diferentes puntos de una ciudad o para conocer la transformación de un sector urbano en el tiempo. En el presente artículo se desarrolla el análisis de la ciudad de Aguascalientes para conocer el estado de desarrollo del hábitat a partir de la información obtenida del Censo Nacional de Población del INEGI del año 2010.

El procedimiento general para obtener los datos cuantitativos es el siguiente:

1. Selección de los polígonos de estudio.

a) Obtención de información geo localizada.

2. Análisis general de información para conocer

a) Densidad poblacional en polígonos.

3. Análisis de Categorías (dimensiones) de sectores seleccionados.

a) Interpretación y vaciado de información geo localizada a tabla.

4. Resultados de Medición.

a) Realización de promedios con base en información geo localizada.

b) Aplicación de fórmula para medir valores de cada dimensión.

c) Obtención de datos finales.

\subsection{Selección de polígonos de estudio}

El primer aspecto que se considera fundamental para obtener la información detallada y significativa fue generar un análisis a nivel manzana. Por lo anterior, para poder encontrar los puntos urbanos más desarrollados y menos desarrollados desde la perspectiva del IDeHa en la ciudad de Aguascalientes, se parte de un análisis de costos de terreno con la información catastral reciente del Gobierno de Aguascalientes.

Se determinó que la zona con mayor costo es: la colonia del Campestre (ver Figura 2).

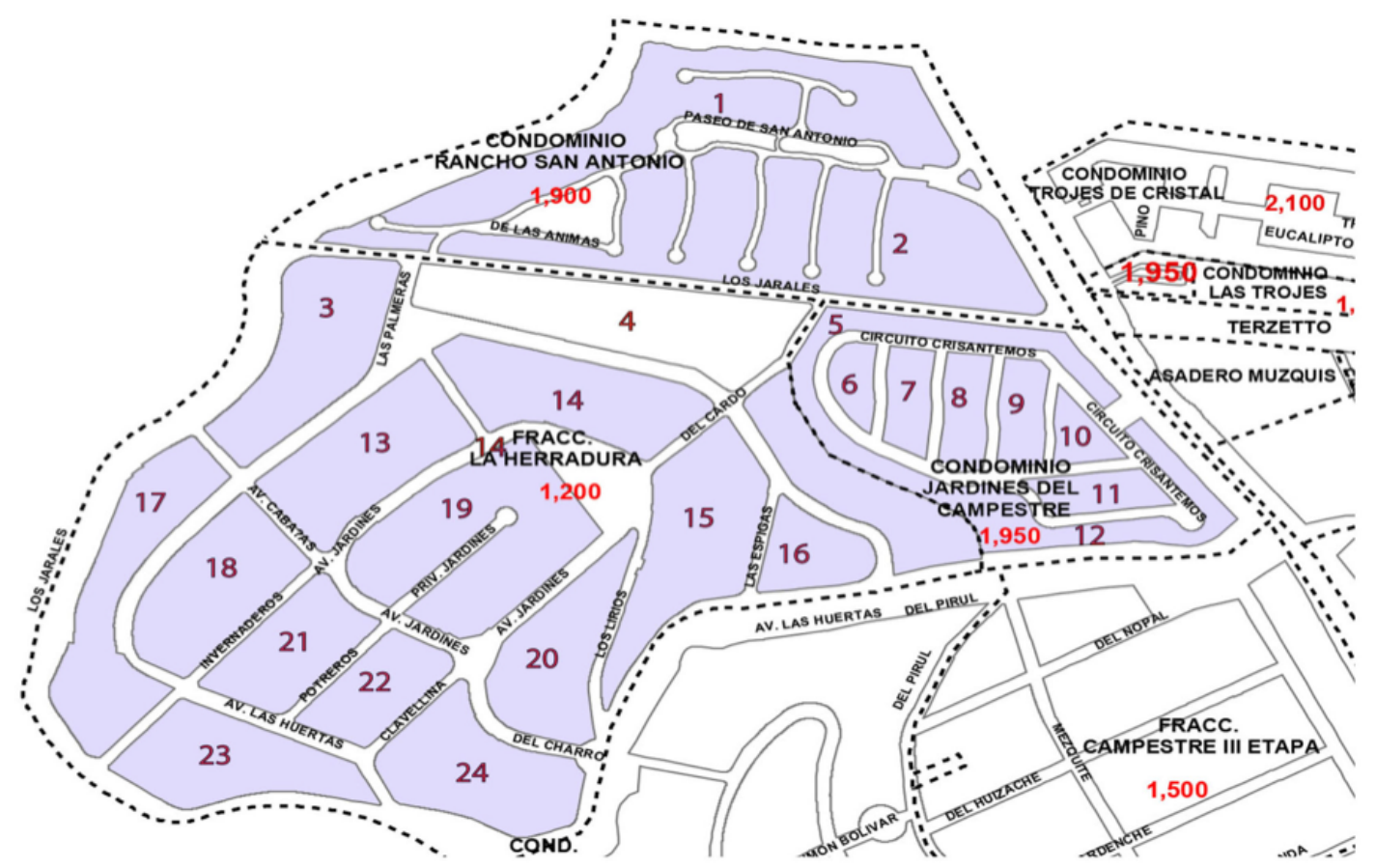

Figura 2. Plano catastral de la zona Campestre. Elaboración propia a partir de información de Gobierno del Estado de Aguascalientes (2010).

El costo por metro cuadrado más alto de la ciudad de acuerdo al catastro correspondiente al periodo de análisis es de: $\$ 1950$ pesos (Gobierno del Estado de Aguascalientes, 2010). 
La zona urbana en Aguascalientes con menor costo, según los datos del catastro, es la colonia de: Los Pericos (ver Figura 3). El costo por metro cuadrado es de: \$550 pesos.

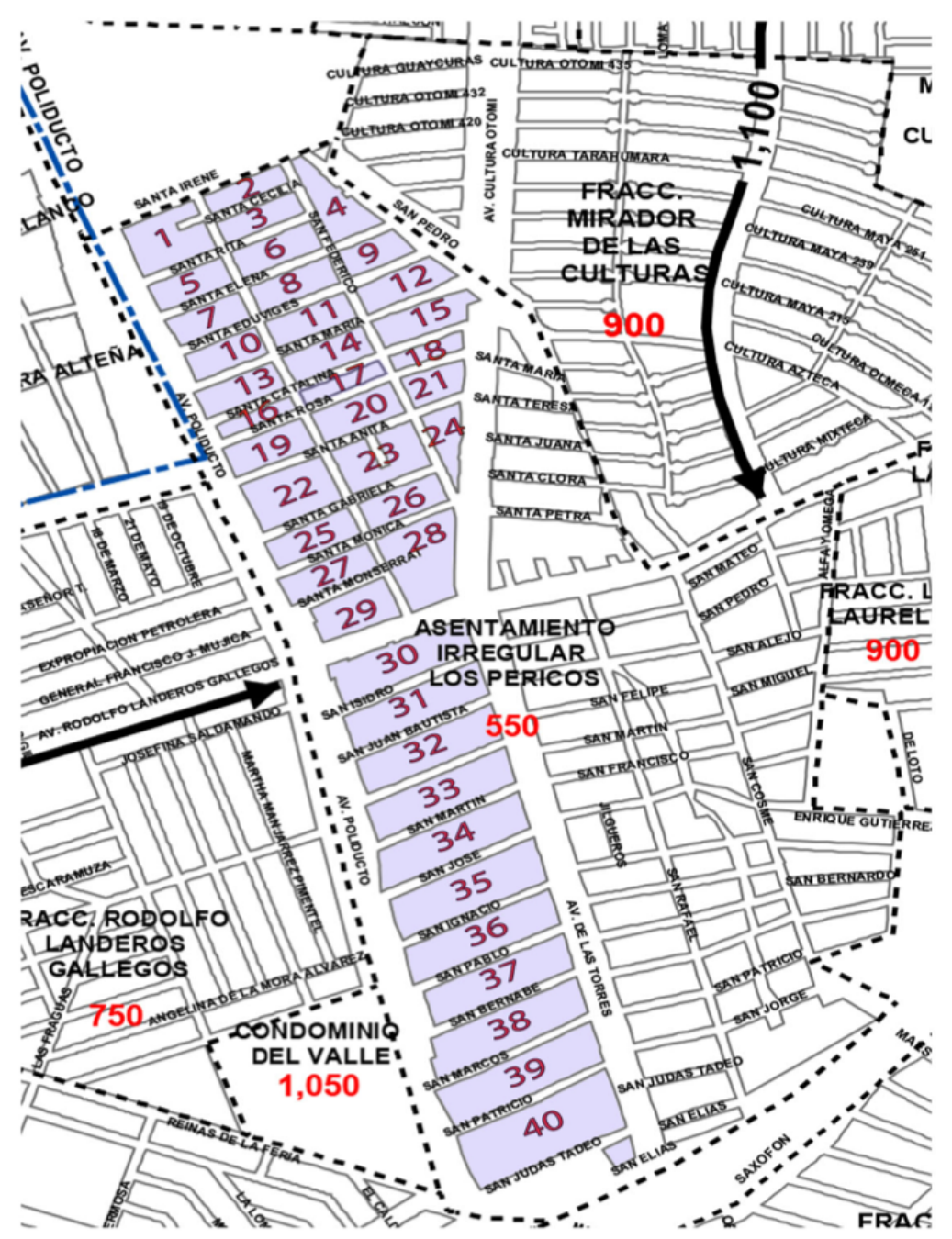

Figura 3. Plano catastral de la zona Los Pericos. Elaboración propia a partir de información de Gobierno del Estado de Aguascalientes (2010).

Para tener un punto de control y verificar que los datos producidos tengan una correspondencia, se decidió tener un punto intermedio en el costo del catastro de la ciudad de Aguascalientes. El costo promedio es de $\$ 1100$ pesos. Esta zona es la colonia Casa Blanca (ver Figura 4 ).

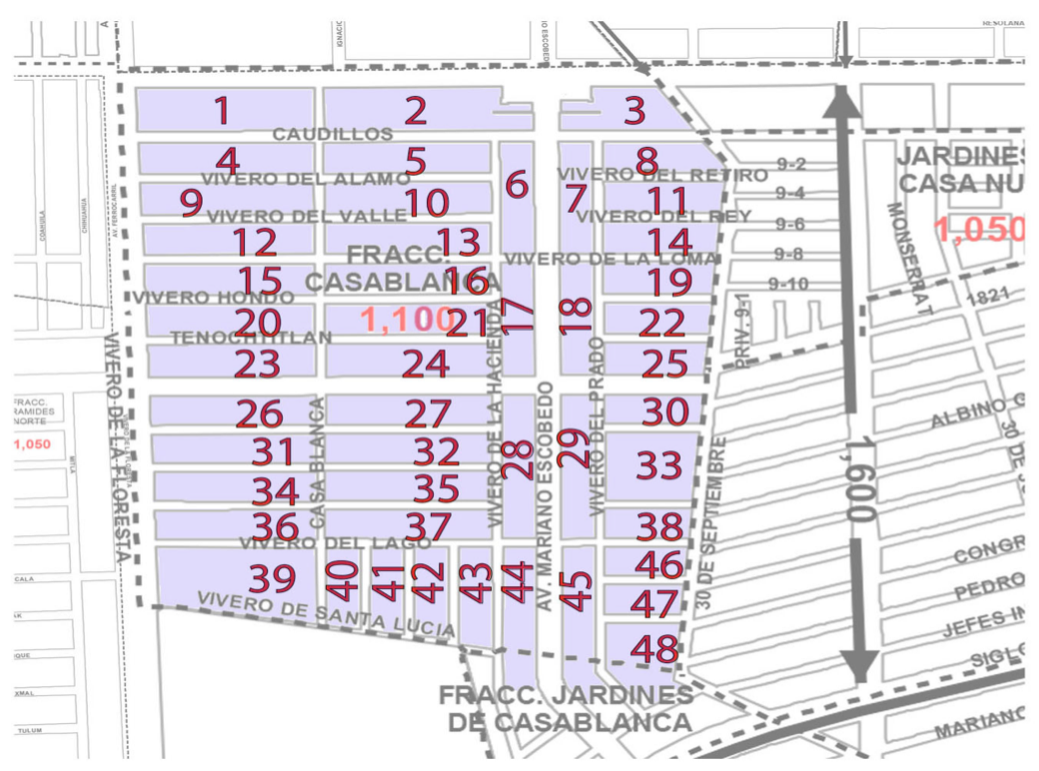

Figura 4. Plano catastral de la zona Casa Blanca. Elaboración propia a partir de información de Gobierno del Estado de Aguascalientes (2010). 
La zona de el Campestre se encuentra ubicada al norte de la ciudad de Aguascalientes. Se asevera que la parte norte de la ciudad tiene un gran equipamiento comercial y deportivo (como un club privado con campo de golf en el Campestre).

La colonia de los pericos se encuentra en el oriente de la ciudad de Aguascalientes. Esta zona se caracteriza por vivienda de interés medio y social.

En el siguiente plano (ver Figura 5) se muestra la localización de las tres zonas de estudio dentro de la mancha urbana de Aguascalientes. La zona del Campestre se encuentra ubicada en la parte superior izquierda, Los Pericos se encuentran en la zona central de lado derecho y Casa Blanca se encuentra ubicada en el punto marcado en el sur de la ciudad.

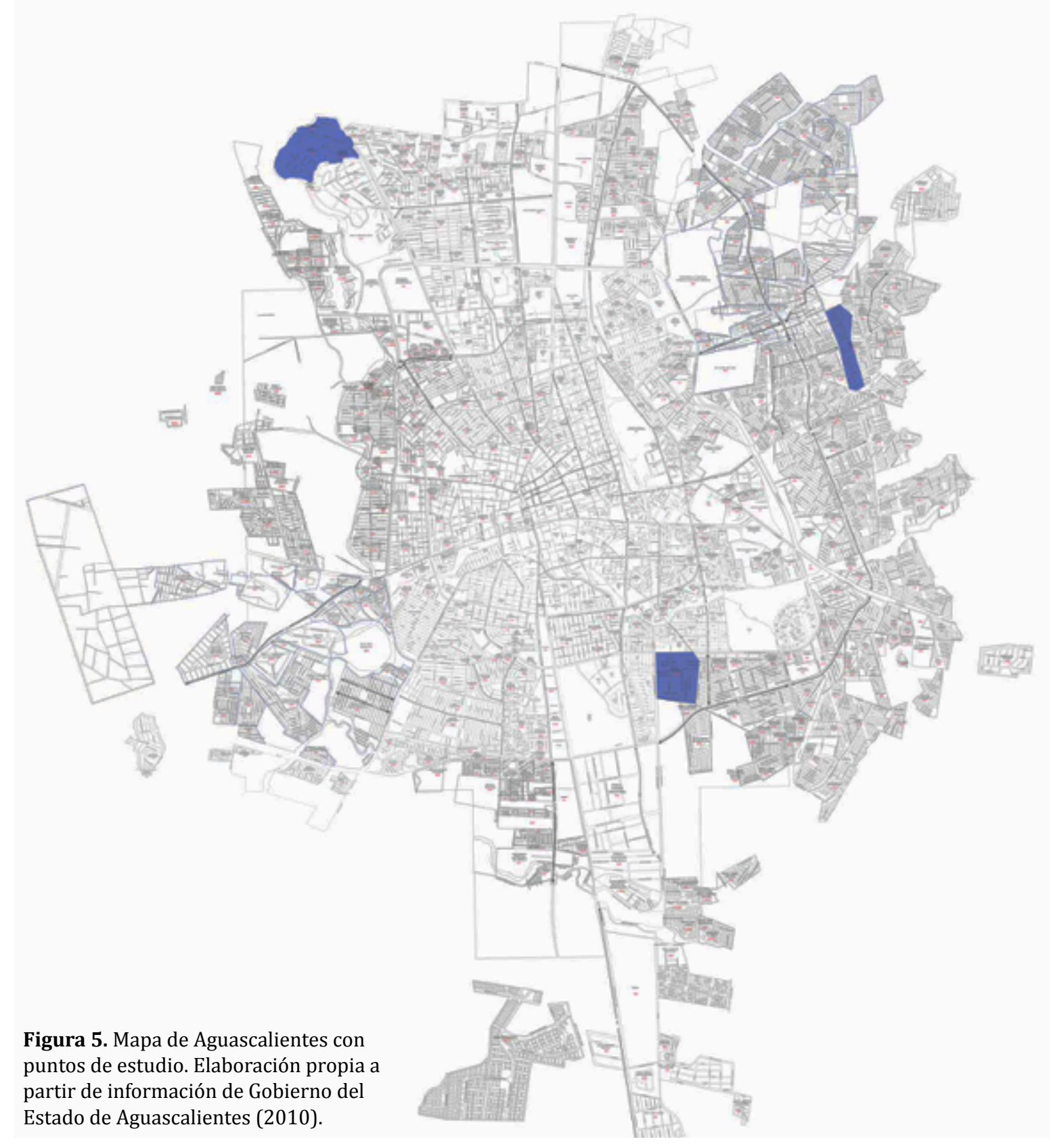

\subsection{Análisis general de información para conocer: Densidad poblacional en polígonos}

Después de tener los tres puntos principales de medición, se hace un análisis de la densidad poblacional en cada una de las zonas. 
Se utilizó el programa QGIS para obtener mapas detallados de información geo referenciada. La densidad se considera un aspecto muy importante para la revisión del IDeHa.

A continuación se muestra la información del número de habitantes por manzana de la Ciudad de Aguascalientes.

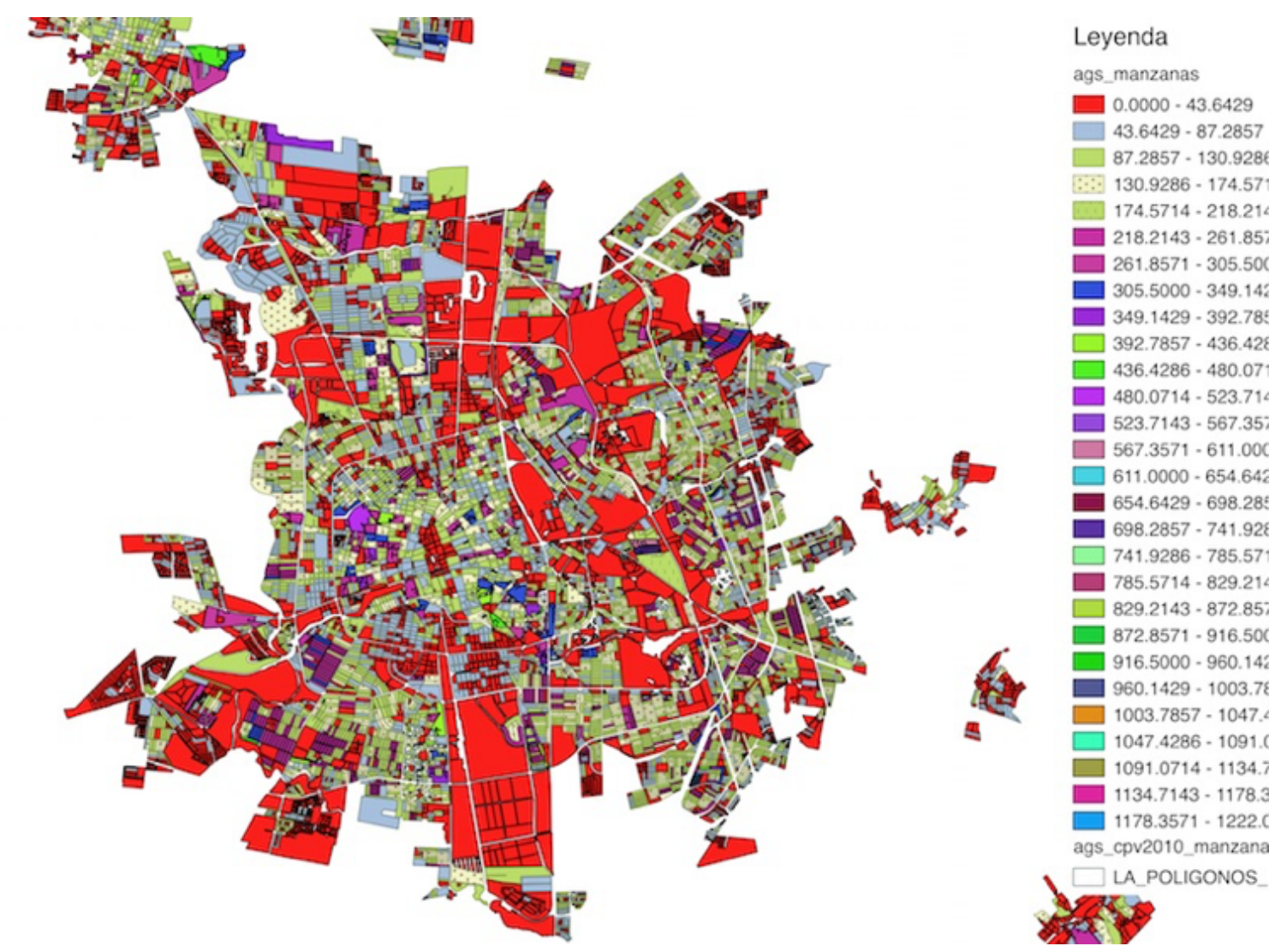

Figura 6. Mapa de densidad poblacional de Aguascalientes. Número de habitantes por manzana. Elaboración propia a partir de datos de INEGI (2010).

Para obtener el número de habitantes por manzana, se hace la relación de: Número de habitantes de la superficie de cada manzana identificada. Se obtiene la siguiente relación del análisis de los polígonos seleccionados:

1. Campestre: 4 habitantes por hectárea (la zona con menor densidad).

2. Los Pericos: 381 habitantes por hectárea (la zona con mayor densidad).

3. Casa Blanca: 150 habitantes por hectárea (promedio).

A continuación se muestran los polígonos de estudio con los datos a nivel manzana en mapa por colonia (columna central), el número de habitantes por manzana (columna derecha) y su relación con el catastro (columna izquierda).

\subsubsection{Polígonos y Análisis}

La Figura 7 muestra el Mapa de puntos de estudio. Superior: Campestre, Intermedio: Los Pericos, Inferior: Casa Blanca. Se trata de elaboración propia (de los autores) a partir de información obtenida de: Gobierno del Estado de Aguascalientes (2010) e INEGI (2010). 


\section{POLIGONOS DE ANALISIS}
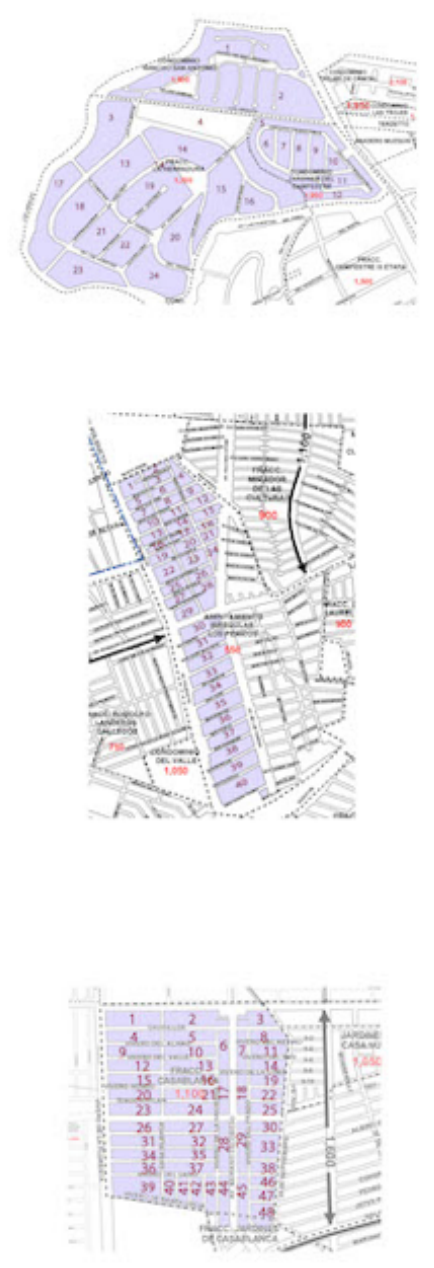
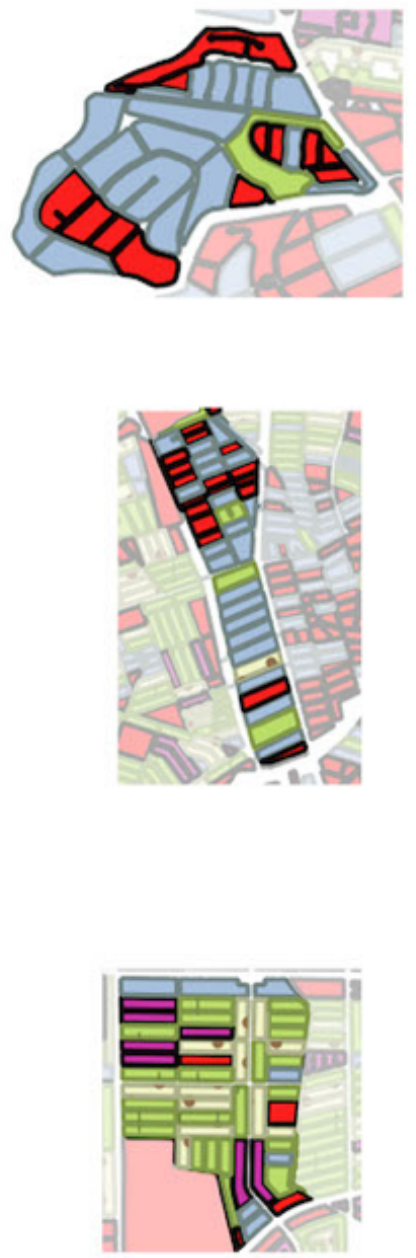

Leyenda

ags_manzanas

$0.0000-43.6429$

$43.6429 \cdot 87.2857$

$87.2857-130.928 t$

$130.9286-174.57$

$174.5714-218.21 /$

$218.2143-261.85$

261.8571 - 305.506

$305.5000-349.14$ ?

$349.1429-392.78$

$392.7857 \cdot 436.42 \xi$

436.4286 - $480.07^{\circ}$

$\square 880.0714 \cdot 523.71$

523.7143 - 567.35:

$\square 67.3571 \cdot 611.00 \mathrm{C}$

$\square 611.0000 \cdot 654.64 \varepsilon$

654.6429 - 698.28:

$698.2857-741.92 \xi$

741.9286 - 785.57 .

$785.5714 \cdot 829.214$

829.2143 - 872.85:

872.8571 - $916.50 \mathrm{c}$

$916.5000 \cdot 960.14$;

$960.1429 \cdot 1003.7 \xi$

$1003.7857-1047$.

1047.4286 - 1091.C

$1091.0714-1134.7$

$1134.7143-1178.3$

1178.3571 - $1222 . c$

ags_cpv2010_manzana

$\checkmark \square$ LA_POLIGONOS

Figura 7. Mapa de puntos de estudio. Superior: Campestre, Intermedio: Los Pericos, Inferior: Casa Blanca. Elaboración propia a partir de información de Gobierno del Estado de Aguascalientes (2010) e INEGI (2010).

\subsection{Análisis de Categorías (dimensiones) de sectores seleccionados}

Posteriormente, se realiza un mapa para cada una de las categorías que se miden dentro del índice (ver Figura 8).

A continuación se muestra el indicador de "Población analfabeta de la categoría POBLACIÓN", para ejemplificar lo que se realizó para cada uno de los indicadores de las 3 categorías:

Porcentaje de población de 15 años y más analfabeta: Personas de 15 a 130 años de edad que no saben leer ni escribir por cada 100 personas de 15 a 130 años de edad.

Se realizó el tratamiento para los 17 indicadores de las 3 categorías (o indicadores): POBLACIONAL, VIVIENDA Y CONECTIVIDAD.

Con la información de todos los indicadores se analizaron los puntos de estudio y se hicieron los siguientes mapas de las zonas específicas (Figuras 9, 10 e 11): 


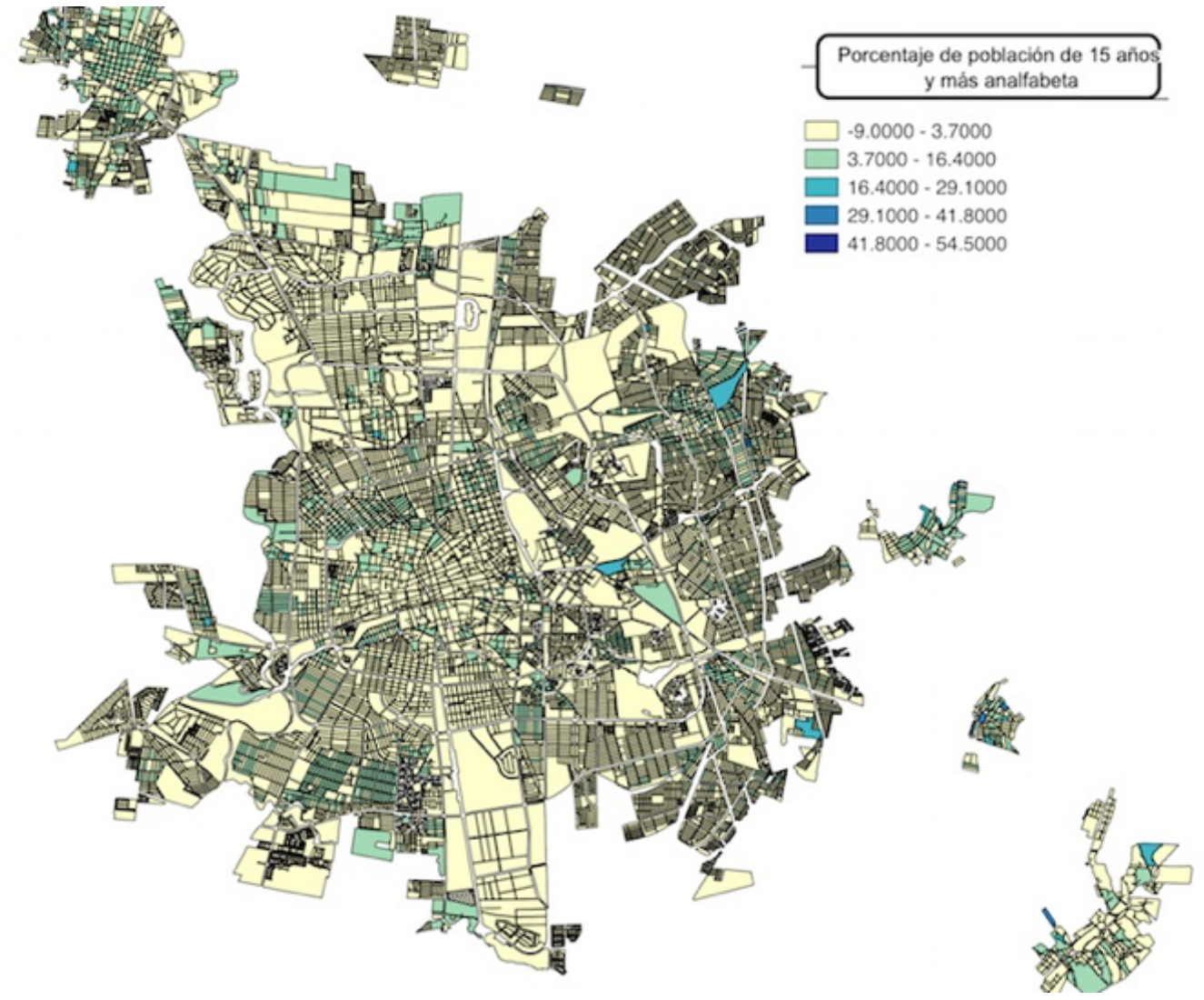

Figura 8. Mapa de población de 15 años o más analfabeta. Elaboración propia a partir de información de Gobierno del Estado de Aguascalientes (2010) e INEGI (2010).
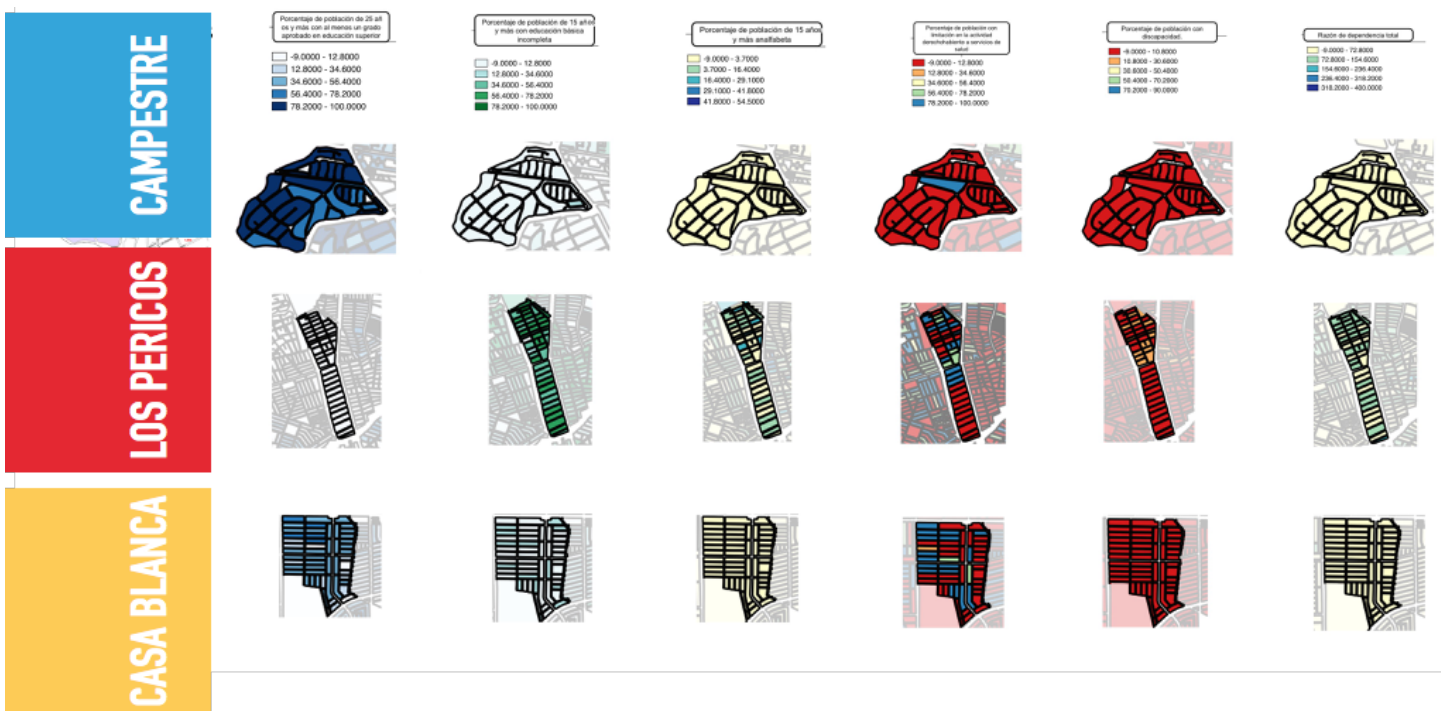

Figura 9. Mapas de análisis de indicadores de estudio de la dimensión: población. Elaboración propia a partir de información de Gobierno del Estado de Aguascalientes (2010) e INEGI (2010). 


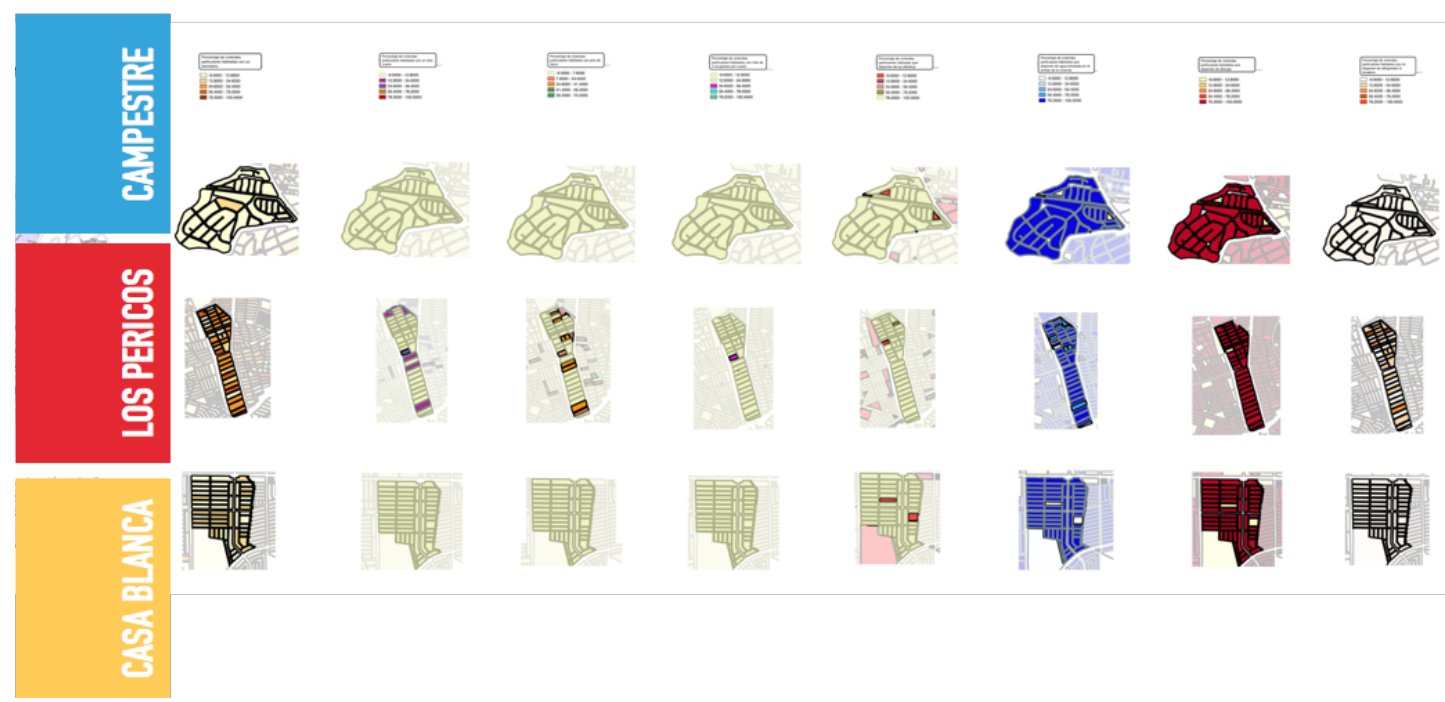

Figura 10. Mapas de análisis de indicadores de estudio de la dimensión: vivienda. Elaboración propia a partir de información de Gobierno del Estado de Aguascalientes (2010) e INEGI (2010).

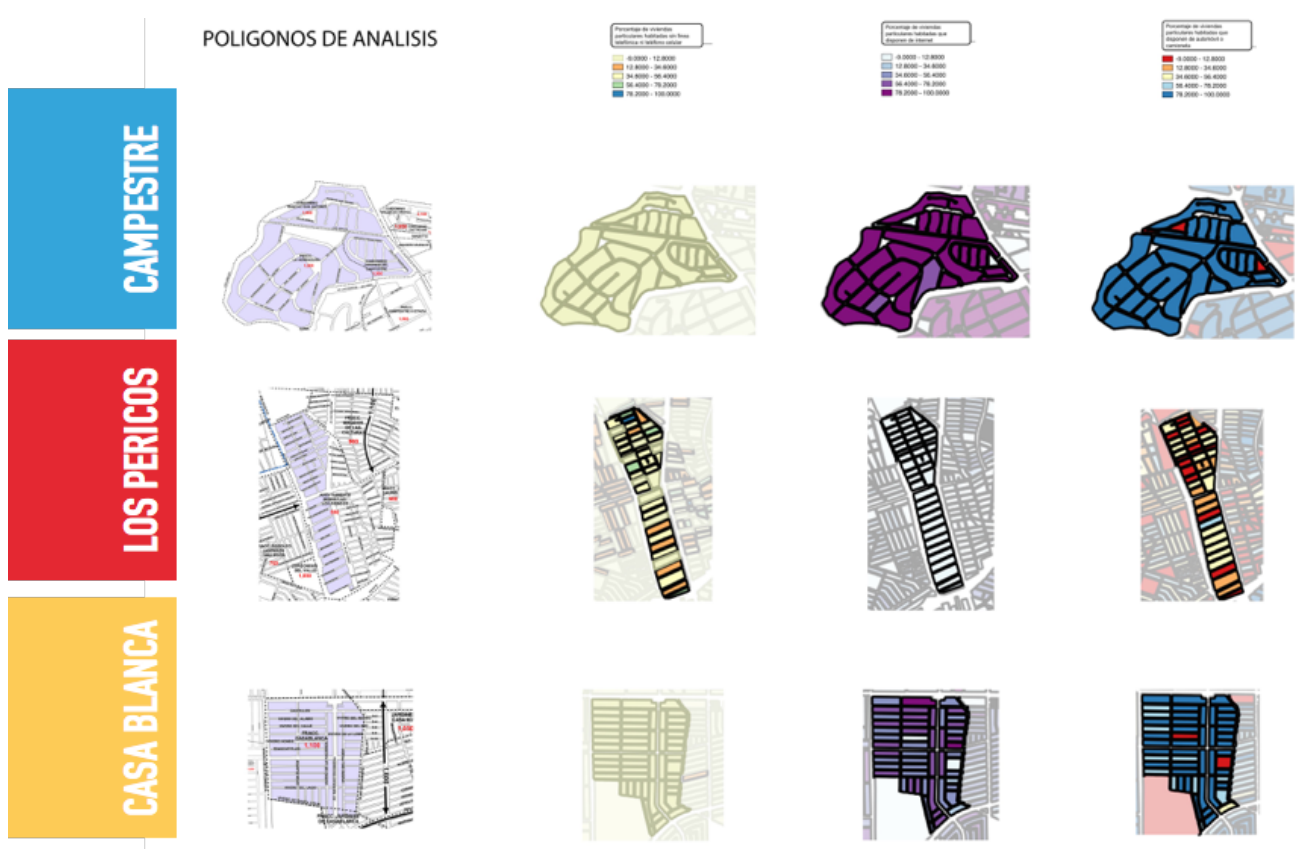

Figura 11. Mapas de análisis de indicadores de estudio de la dimensión: conectividad. Elaboración propia a partir de información de Gobierno del Estado de Aguascalientes (2010) e INEGI (2010).

Posteriormente, se hace una interpretación de cada uno de los datos por manzana y se le asigna un carácter numérico de acuerdo al rango en el que se encuentra la información de cada manzana.

Por ejemplo: Para el punto de análisis de El Campestre para la dimensión de POBLACIÓN, se asignó un número a cada manzana de la colonia y se consideró el rango inicial y final del mapa generado en QGIS para obtener el promedio numérico de cada manzana.

Así, para el primer indicador de la primera categoría tenemos un rango inicial de: $78.2 \%$ y uno final de: $100 \%$. El promedio es de: $89.1 \%$, por lo tanto el indicador tiene un valor del promedio: $89.10 \%$.

El procedimiento se realiza para todos los datos de cada manzana de estudio. El proceso mostrado con anterioridad se realiza para las tres categorías ( y para las tres zonas de estudio) (ver Figuras 12 y 13). 


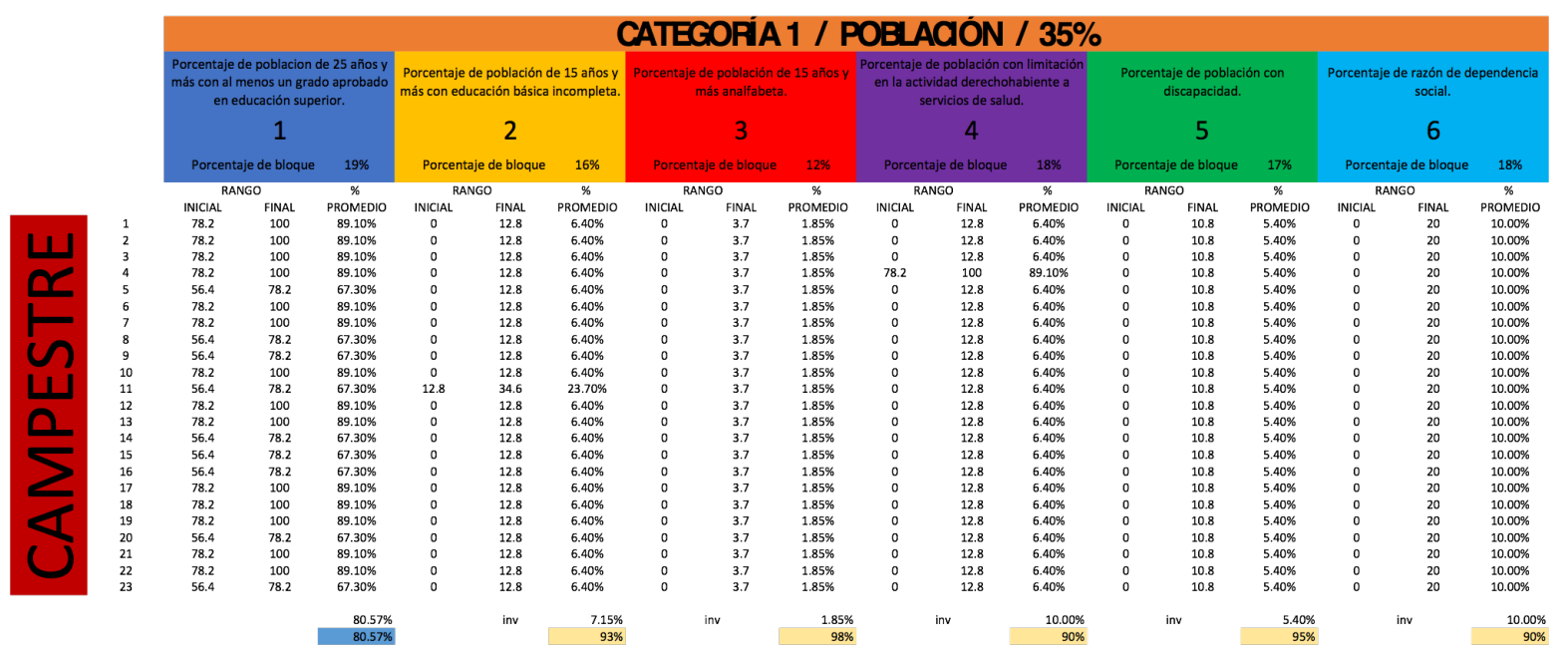

Figura 12. Información de la colonia el Campestre para la categoría de: POBLACIÓN. Elaboración propia a partir de información de Gobierno del Estado de Aguascalientes (2010) e INEGI (2010).

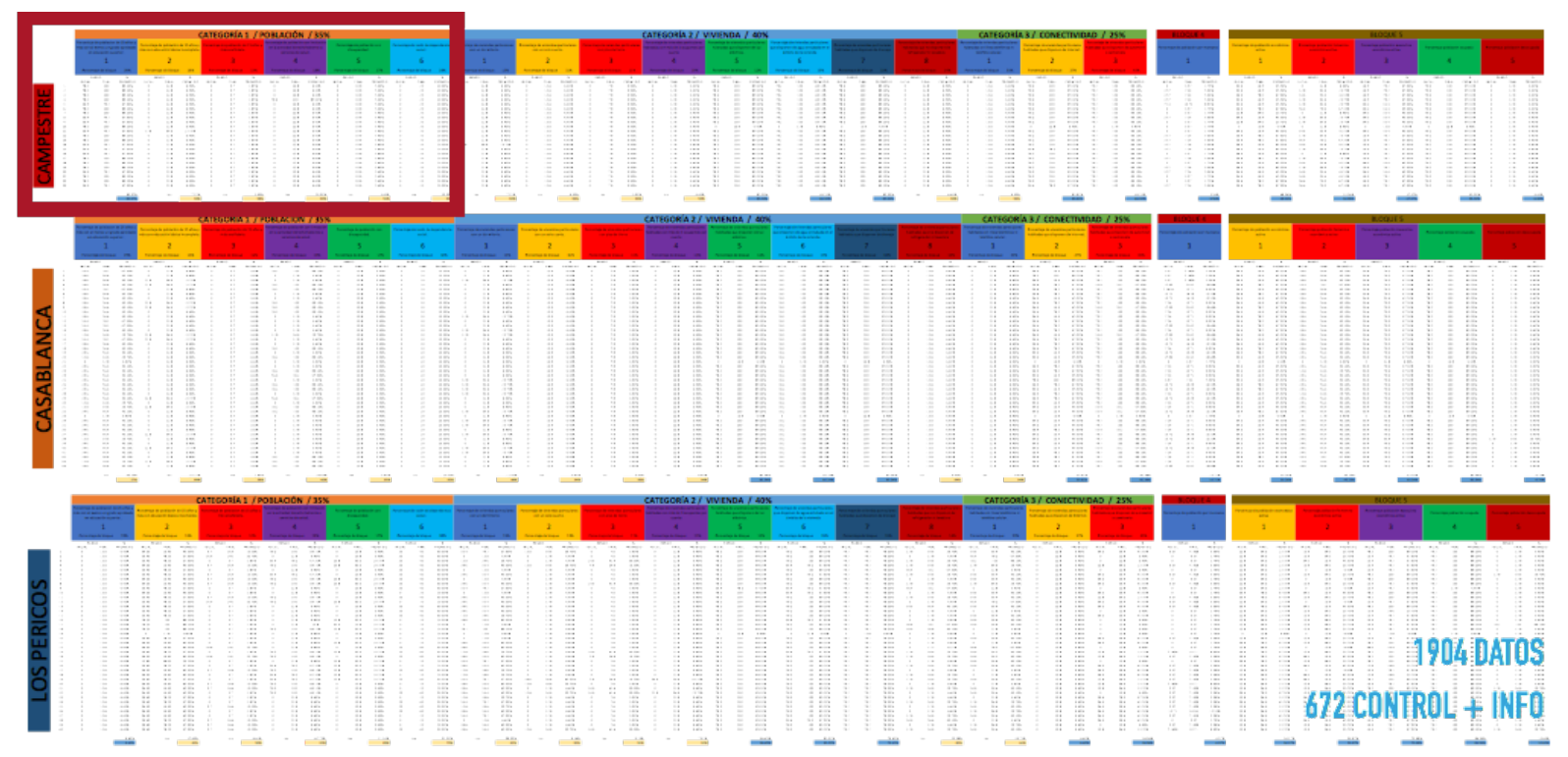

Figura 13. Información de los tres puntos de estudio y las tres dimensiones que conforman el Índice. El recuadro en rojo representa la Figura 12. Elaboración propia a partir de información de Gobierno del Estado de Aguascalientes (2010) e INEGI (2010).

\subsection{Resultados de medición}

Con la tabla completa, se obtienen 1904 datos, 672 datos de control para el presente estudio. También hay posibilidades de obtener otros datos con carácter informativo para conocer mejor los aspectos sociodemográficos de las manzanas que se han estudiado.

Después de la obtención de todos los datos, es necesario calcular el índice a partir de una correspondencia válida de cada uno de los factores o indicadores que comprende la tabla.

La función que se ha utilizado para dicho fin es la siguiente:

$$
I D H=f\left(x_{1 t} x_{2 t} x_{3 t} x_{n t} \ldots\right)
$$

Siempre y cuando IDH $\leq 1$ y es significativo

Aplicando la función (1) a los datos obtenidos de las tres dimensiones, obtenemos la siguiente tabla (Figura 14): 


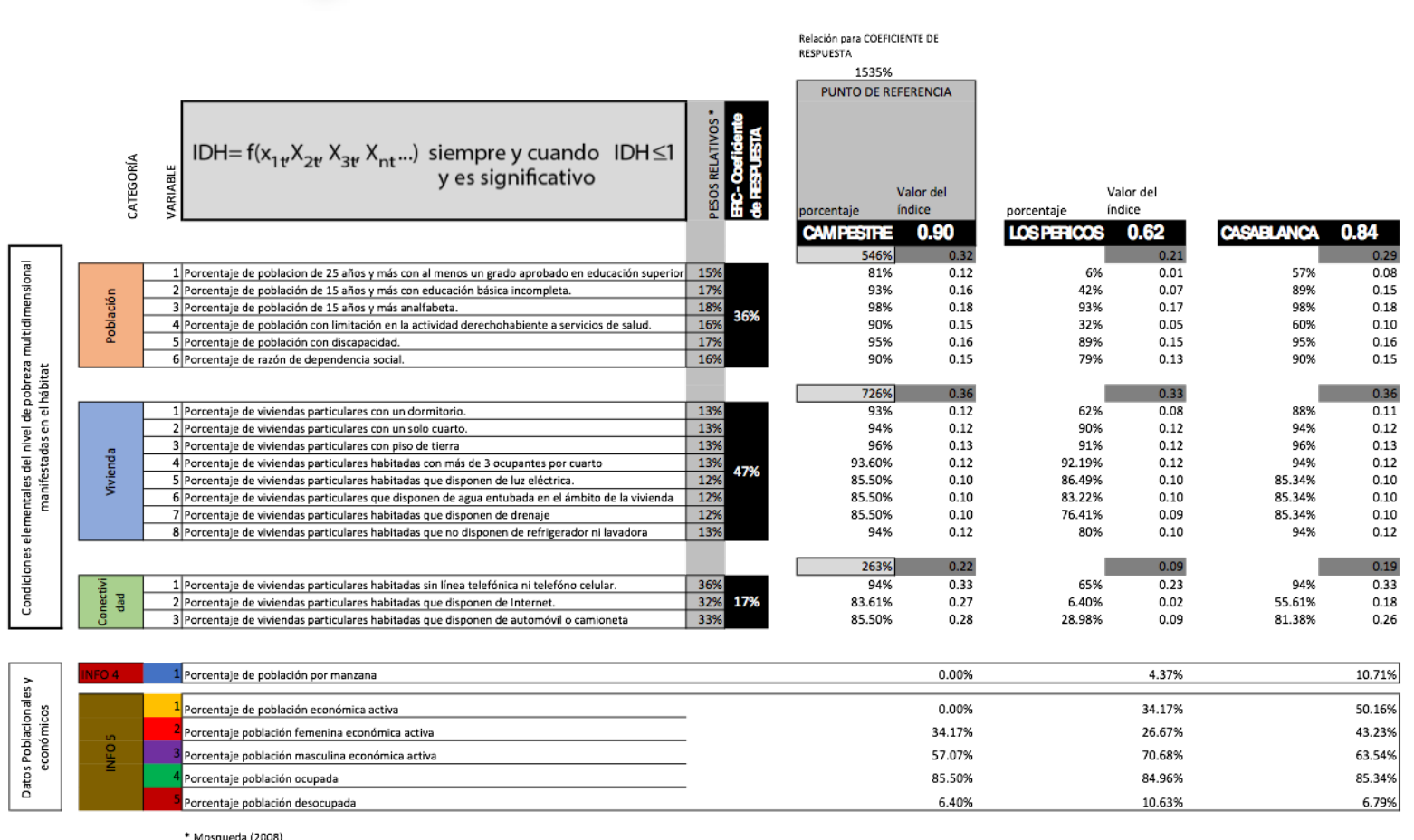

Figura 14. Tabla para la obtención de datos para cálculo de Índice para el Desarrollo del Hábitat. Elaboración propia a partir de información de Gobierno del Estado de Aguascalientes (2010) e INEGI (2010).

Con la Tabla 14, se observa que las variables relacionadas con la POBLACIÓN, tienen un peso del $36 \%$ del total del valor del índice. La VIVIENDA tiene un peso de $47 \%$ y la CONECTIVIDAD en un $17 \%$.

La evaluación del Índice para el Desarrollo del Hábitat indica que los sectores urbanos medidos que tengan resultados de uno o cercanos a uno, son aquellos que se encuentran con las más altas características de desarrollo del hábitat. Entre más se aleje el valor de uno hacia valores inferiores, indica que el lugar analizado tiene menores características de desarrollo del hábitat.

Para cada uno de los sectores analizados de la ciudad de Aguascalientes, tenemos el siguiente promedio considerando las tres categorías:

1. Campestre: 0.90

2. Los Pericos: 0.62

3. Casa Blanca: 0.84

Cada uno de los valores que se muestran en la tabla, representan una oportunidad para mejorar las condiciones del hábitat de los sectores que se encuentran menos desarrollados.

\section{Conclusiones}

El Índice para el Desarrollo del Hábitat es una herramienta que puede facilitar la comprensión de la situación urbana y su desarrollo. La herramienta tiene una utilidad para hacer análisis comparativos de diferentes sectores urbanos, así como de un mismo lugar en el tiempo.

Con el IDaHa de la ciudad de Aguascalientes, se comprueba que la ciudad tiene un contraste muy marcado entre las zonas con más desarrollo urbano como la colonia el Campestre y las zonas menos desarrolladas como la colonia Los Pericos.

A partir de los datos obtenidos, se abre la posibilidad de analizar con mayor profundidad las características, las causas y las relaciones que hay en esta diferencia socioeconómica y de calidad del hábitat entre los diferentes puntos urbanos de una misma ciudad. 
Así, cada uno de los valores que se muestran en la tabla, representan una oportunidad para mejorar las condiciones del hábitat de los sectores que se encuentran menos desarrollados.

La aplicación del índice podría coadyuvar en la elaboración de planes de desarrollo, para focalizar esfuerzos en el planteamiento de estrategias de mejoramiento para determinadas zonas de la ciudad, con la intención de superar el riesgo o vulnerabilidad de sus habitantes.

\section{Referencias}

Bauman, Z. (1999). Trabajo, consumismo y nuevos pobres. Barcelona: Editorial Gedisa.

Bauman, Z. (2005). Vidas desperdiciadas. La modernidad y sus parias. Barcelona: Editorial Paidós.

Bauman, Z. (2008). Comunidad. Madrid: Editorial Siglo XXI.

Bauman, Z. (2013). La vida líquida. Barcelona: Editorial Planeta.

Brebbia, C., \& Pulselli, R. (Ed.). (2014). Eco-Architecture V. Harmonisation between Architecture and Nature. United Kingdom: Wessex Institute of Technology Press.

Boff, L. (2001). Ética planetaria desde el Gran Sur. Madrid: Editorial Trotta.

Gobierno del Estado de Aguascalientes (2010). Instituto Catastral. Consultado en: http:// www.aguascalientes.gob.mx/sefi/catastro

Hardt, M., \& Negri, A. (2000). Empire. Cambridge, Massachussets: Harvard University Press.

Instituto Nacional de Estadística y Geografía (INEGI). (2011). Encuesta Nacional de Ocupación y Empleo (ENOE). Consultado en: $\underline{\text { http://www.inegi.org.mx }}$

Encuesta Nacional de Población, 2010. (2010). Consultado en: $\underline{\text { http://www.inegi.org.mx }}$

Latouche, S. (2009). Decrecimiento y Posdesarrollo: El pensamiento creativo contra la economía del absurdo. Mataró (España): Intervención Cultural.

Max-Neef, M., Elizalde, A., \& Hopenhayan, M. (1993). Desarrollo a escala humana. Una opción para el futuro. Santiago: Centro de Alternativas de Desarrollo, CEPAUR.

Sen, A. (2000). Desarrollo y Libertad. Buenos Aires: Editorial Planeta.

Stiglitz, J. (2002). El malestar de la Globalización. Madrid: Editorial Punto de lectura.

Stiglitz, J. (2012). El precio de la desigualdad. Madrid: Editorial Taurus. 